\title{
POST-COVID-19 REACTIVE ARTHRITIS - CASE REPORT
}

Verônica Palmiro da Silva e Lima ${ }^{1, \star}$, Juliana Carollyne Amorim${ }^{1}$, Ariane Caroline Mota Souza Silva ${ }^{1}$, Ana Maria dos Santos Oliveira ${ }^{1}$, Ayrton Senna do Brasil Amaral Alves ${ }^{1}$, Karla Viana Rezende $^{1}$, Sarah Regina Alves de Alencar ${ }^{1}$, Walquiria Santos Davi ${ }^{1}$, Guilherme Sampaio Silva ${ }^{1}$

1.Universidade do Estado de Mato Grosso, Cáceres (MT), Brazil.

*Corresponding author: veropalmiro@hotmail.com

\section{BACKGROUND}

The triggering mechanisms of the sequelae of COVID-19 are still unknown in many cases, but as it is a pandemic, case reports for the exchange of experiences are important for the development of coping strategies for these cases, since such consequences may occur on a large scale. This work aims to contribute to the scientific community, demonstrating an autoimmune picture probably induced by SARS-CoV-2 and a successful therapeutic strategy.

\section{CASE REPORT}

Female patient, 87 years old, hypertensive, sought the rheumatology service complaining of arthralgia in the right ankle, knee, wrist and shoulder. These symptoms appeared about 15 days after SARS-CoV-2 infection. On physical examination, she presented synovitis in the right knee and ankle. Brought an image and a chest tomography report with findings related to COVID-19, such as areas of ground glass and consolidation throughout the both lung lobes, with thickening of the bronchial walls, affecting $<50 \%$ (findings related to COVID-19). Laboratory tests showed a significant increase in markers of inflammatory activity with ESR $=90$ $(\mathrm{VR}<20)$ and $C R P=18(\mathrm{VR}<6)$; however, with negative or insignificant results in the autoantibody screening, it was observed: $F R<8$, anti-CCP negative, 1/80 nuclear ANA dense fine speckled and absent HLA-B27. Other biochemical tests of blood and urine showed no changes, as well as negative viral serology for hepatitis B, C and HIV. Having as a diagnostic hypothesis post-COVID-19 reactive arthritis, prednisone $20 \mathrm{mg} /$ day was prescribed for 30 days. On return, the patient already reported no pain and had $\mathrm{ESR}=20$ and $\mathrm{CRP}<6$, thus weaning from prednisone was performed.

\section{CONCLUSION}

The case presented above reaffirms the hypotheses recently raised by the scientific community, which suggest that SARS-CoV-2 has the ability to induce systemic autoimmune diseases, in this case reactive arthritis was the pathology triggered.

\section{KEYWORDS}

Reactive arthritis, SARS-CoV-2, Covid19, Autoimmune. 